\title{
The Analysis of Power System Static Stability based on Matlab/Simulink Yang Cai ${ }^{1,}$, Shiwei Su ${ }^{1, b}$ \\ ${ }^{1}$ College of Electrical Engineering \& New Energy, China Three Gorges University, China \\ acaiyang1019@sina.com, bssw@ctgu.edu.cn
}

Keywords: power system, stability,disturbance,simulation

Abstract. In this paper, the power system blockset in Matlab/Simulink is applied to build a one-machine infinite-bus system to simulate the actual power system. The power system static stability will be discussed according to the severity of disturbance. Also simulation analyses and improvement measures will be made. And conclusion can be drawn from the paper that power system static stability would be influenced by the exertion of Power System Stabilizer and the excitation voltage gain.

\section{Introduction}

With the growth in economic and societal energy demand, power systems are essential. A power system is a complex dynamic system, which must ensure energy quality and quantity. A power system, however, always faces constant disturbances that can occur at random locations and times. Power disturbances fall into two types: large and small. Large disturbance occur due to short circuit faults or protect delay device malfunctions. The power system is stable if it can restore balance when the disturbance is removed. The restored balance may be the original operation state or a new stable state. The static stability of the power system can be restored to the original stable state (or to maintain the gradually approaching stability) after the disturbance from a normal operating state.

\section{Model building of one-machine infinite-bus system}

The power system blockset (PSB) and Simulink based on Matlab are introduced to build a model one-machine infinite-bus system (OMIB). The 'Synchronous Machine pu Standard','Excitation system,' 'Three-Phase Transformers,' 'Distributed Parameters Line,' 'Three-Phase Parallel RLC Loads,' 'Three-Phase Source,' 'Three-Phase V-I Measurements' and 'Scope' are applied in the model of one-machine infinite-bus system. A generator of parallel operation with a transformer and transmission lines is connected in an infinite bus system. Such a system can be considered to have infinite power, constant frequency and constant voltage.

\section{Simulation analysis of power system static stability}

\section{Model building of power system static stability}

The parameters of generator: $\mathrm{S}_{\mathrm{GN}}=325.5 \mathrm{MV} \cdot \mathrm{A}, \mathrm{P}_{\mathrm{GN}}=300 \mathrm{MW}, \mathrm{U}_{\mathrm{GN}}=10.5 \mathrm{kV}$

The parameters of transformers: $\mathrm{S}_{\mathrm{TN} 1}=360 \mathrm{M} \cdot \mathrm{VA}, \mathrm{k}_{\mathrm{T} 1}=10.5 / 242, \mathrm{~S}_{\mathrm{TN} 2}=360 \mathrm{M} \cdot \mathrm{VA}, \mathrm{k}_{\mathrm{T} 2}=220 / 121$

The parameters of transmission line: $1=250 \mathrm{~km}, \mathrm{x}_{1}=0.41 \Omega / \mathrm{km}, \mathrm{r}_{\mathrm{l}}=0.07 \Omega / \mathrm{km}$

Operation Conditions: $\mathrm{U}_{0}=115 \mathrm{kV}, \mathrm{P}_{0}=250 \mathrm{MW}, \cos \varphi_{0}=0.95$

Power system static stability is an inherent characteristic of power system. The static stability is related to the structure and parameters of the power system. Therefore the static stability of a power system can be changed by modifying the excitation regulation of synchronous generator and the reactance parameters of line.

In this paper, the power angle, rotor speed, and electromagnetic power of the synchronous generator are measured to determine system stability. The power system is modeled with the "phase" Powergui module. The synchronous generator is a PV node, whose terminal voltage is $10.5 \mathrm{kv}$ and active power is $260 \mathrm{MW}$. The simulation algorithm used is the Ode23tb with a 10 second simulation time. The model is shown in the Figure 1. 


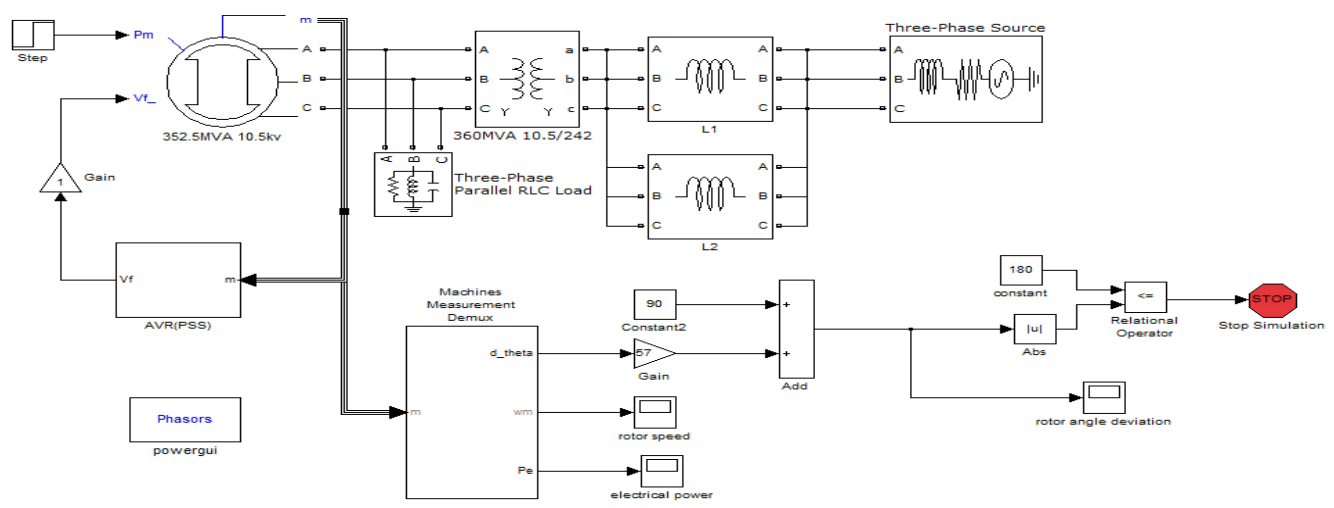

Figure 1 Model of OMIB System

\section{Simulation waveform analysis of power system static stability}

\section{Simulation Analysis of the influence of Automatic Excitation Control System}

In modern power systems, a synchronous generator is equipped with an automatic excitation regulator system. The excitation system of the synchronous generator is introduced into the signal of the direct axis and cross axis. The internal transfer function is compared with the output excitation voltage signal, and the feedback to the generator and the system. When the system is disturbed by less than $10 \%$ of the active power output, the automatic excitation control system of the generator can restore the stability. But if the disturbance increases, the regulation of the excitation system will decrease.

When generator mechanical power input step are Initial: 0.4 (p.u.) and Final :0.44 (p.u.) , electromagnetic power waveform is shown in Figure 2 .

When generator mechanical power input step are Initial:0.4 (p.u.) and Final:0.8 (p.u.) , electromagnetic power waveform is shown in Figure 3 .

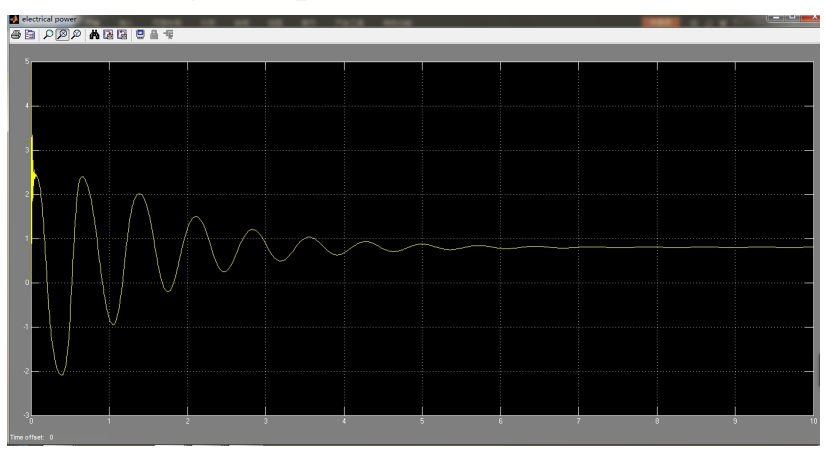

Figure 2 Electromagnetic Power Waveform Disturbance less than $10 \%$

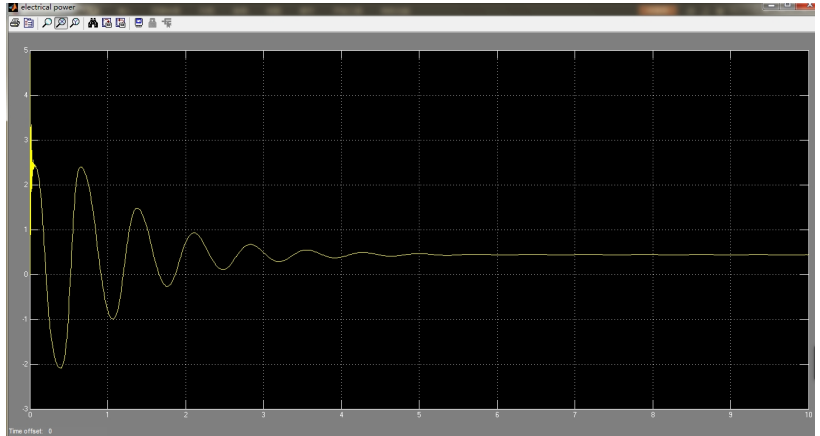

Figure 3 Electromagnetic Power Waveform Disturbance more than $10 \%$

\section{Simulation Analysis of the influence of Power System Stabilizer (PSS)}

The generator excitation system contains a power system stabilizer (PSS), functioning as a sub module of the excitation system, the output of which is a kind of excitation input signal. The basic function of a PSS is to control the excitation of the generator rotor to enhance the damping of the generator rotor by exerting the additional stable signal. In the simulation model, the "Switch Manual" is connected with the PSS.

Generator mechanical power input step is Initial:0.4 (p.u.) Final:0.416 (p.u.) waveform of the rotor speed is shown in the figure. Figure 4 is one without PSS, while Figure 5 is with PSS. 


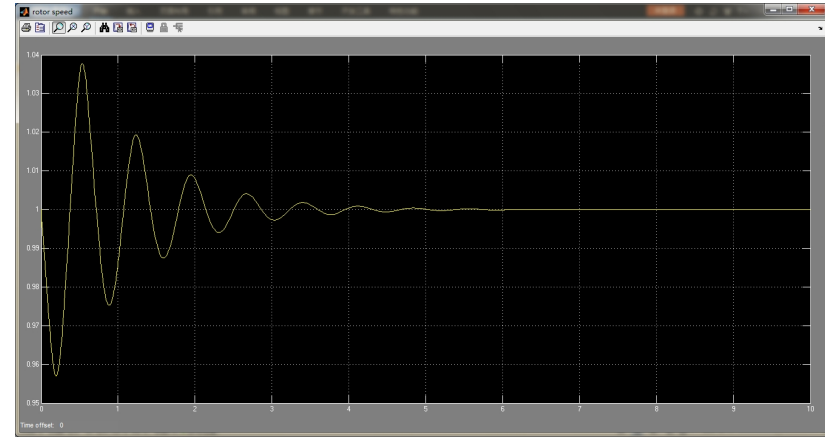

Figure 4 Waveform of the Rotor Speed

With PSS ( small disturbance )

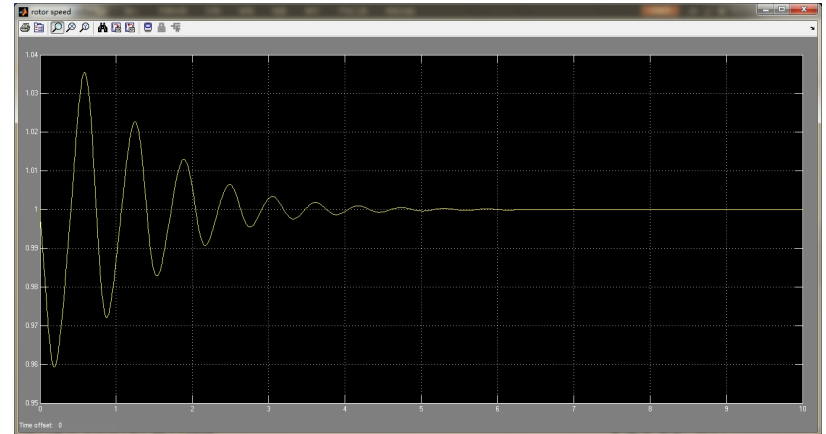

Figure 5 Waveform of the Rotor Speed

Without PSS ( small disturbance )

Generator mechanical power input step is Initial: 0.4 (p.u.) Final: 0.6 (p.u.). The waveform of the rotor speed is shown in the figure. The Figure 6 is one without PSS, while Figure 7 is with PSS.

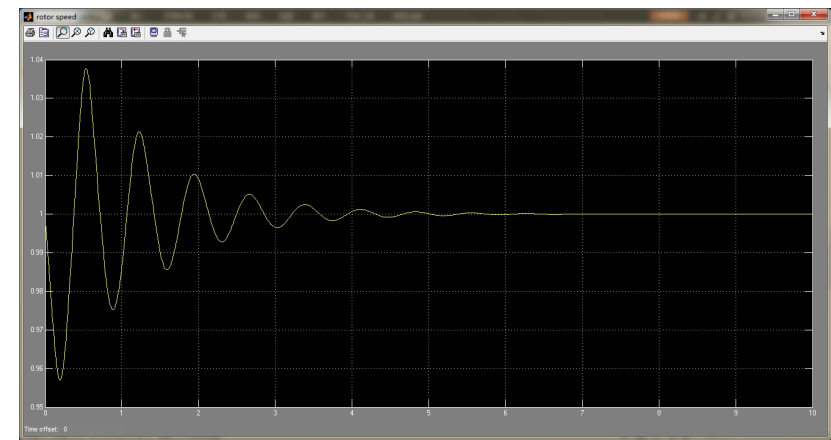

Figure 6 Waveform of the Rotor Speed With PSS ( large disturbance )

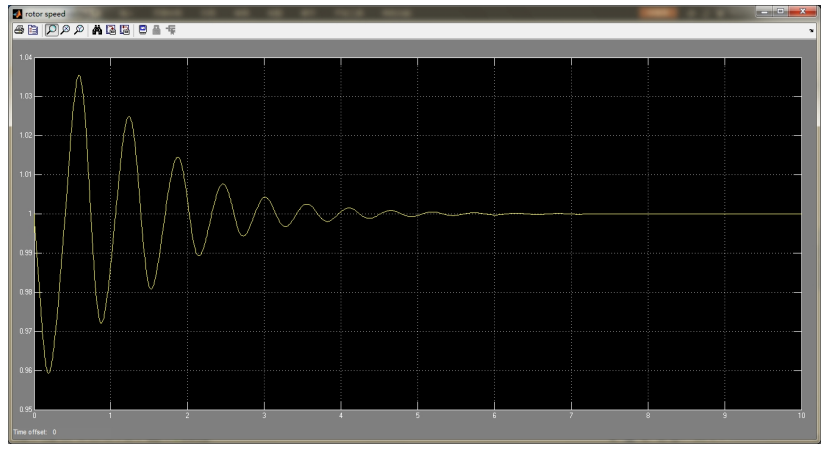

Figure 7 Waveform of the Rotor Speed Without PSS ( large disturbance )

When the output power is $4 \%$, the input PSS can reduce the system stabilization time. When the output power is $50 \%$, the PSS switch is unable to change the stability of the system.

\section{Simulation Analysis of the influence of the Gain of Excitation voltage}

As discussed earlier, the automatic control system of generator and PSS system stabilizer can play a role within a certain range of the disturbance. Additionally, the installation of the excitation voltage gain device (Gain) changes the excitation voltage of a generator to restore the stability.

When the input mechanical power of generator is Initial:0.4 (p.u.) and Final:2 (p.u.), the combined application of 'Gain=1, AVR and PSS' cannot stabilize the system (as is shown in the Figure 8); when the excitation voltage gain is changed to ' Gain $=5$ ', the system can restore stability(as is shown in the Figure 9).

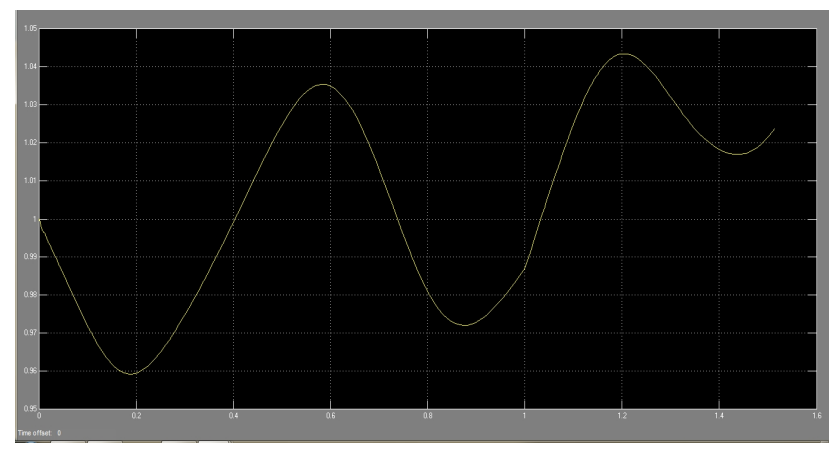

Figure 8 Waveform of the Rotor Speed Gain=1

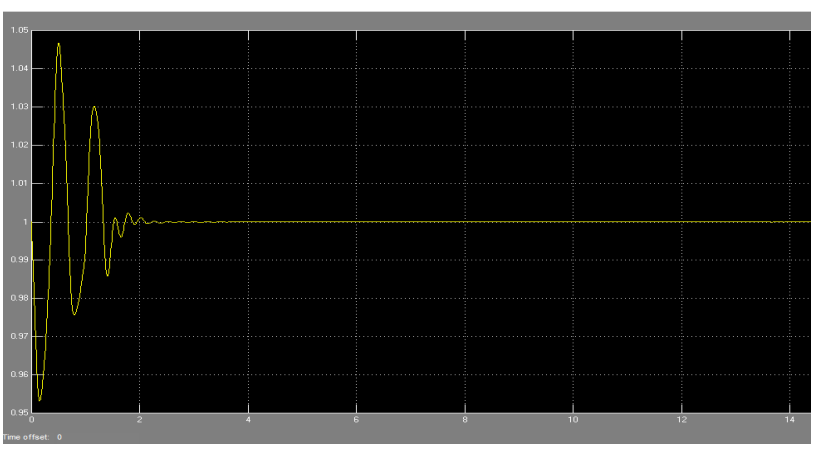

Figure 9 Waveform of the Rotor Speed Gain=5

\section{Simulation Analysis of the influence of serial capacitor}

Series capacitor functions as compensate in OMIB system. When the system is under a 5\% disturbance, the series capacitor can restore system stability without PSS and excitation voltage gain.

Conclusion: The series capacitor can improve the static stability of a power system at a relatively low cost. 


\section{Improvement measures for power system static stability}

The following conclusions can be drawn from the simulation:

(1) Modern power systems are equipped with automatic excitation systems (AVRs), which can restore the stable state when confronting disturbance less than $10 \%$. But when the disturbance increases, the ability to stabilize diminishes.

(2)A small disturbance can be adjusted by the power system stabilizer (PSS). But when the disturbance is too large, switch PSS will not improve the power system stability.

(3) The increase of the excitation voltage gain and combination of PSS can effectively reduce the power system restoration time and improve the static stability of the power system.

(4)The series capacitor in the circuit transmission line can improve the static stability of the power system cheaply.

\section{Conclusion}

In this paper, the Matlab/Simulink is used to build an OMIB system. The system is simulated by small interference for the static stability of power system.

Taking simulation results into consideration, the results are consistent with the relevant theoretical conclusions. From the results, the exertion of power system stabilizer (PSS),especially combined with the increase of excitation voltage gain can effectively reduce the recovery time and improve the stability of the generator.Additionally, series capacitors can function as compensate to improve the static stability of the system.

Matlab/Simulink can be directly used for power system power flow analysis, steady state and transient simulation, and interface flexibility. But the simulation process model is based on ideal assumptions, and the simulation speed needs to be improved. Furthermore the circuit design should be simplified.

\section{References}

[1] Jingxia Liu.Sci-tech Information Development \& Economy.2008.Vol 18. (In Chinese)

Reference to a book:

[2] Daozhi Xia, Power System Analysis, second ed.,China Electric Power Press, 2012. (In Chinese)

[3] Qun Yu, MATLAB/Simulink Power System Modelling and Simulation, China Machine Press,2011. (In Chinese) 